\title{
CONDICIONES LABORALES Y NIVEL DE ESTRÉS EN ENFERMEROS DE UN HOSPITAL PÚBLICO PERUANO
}

\author{
Wilson Sauñe-Oscco ${ }^{1, a}$, Carmen E. Bendezù-Sarcines ${ }^{2,3, a}$. , Olinda Oscco-Torres ${ }^{2,4, a}$.
}

1 Puesto de Salud Santa Ana de Aucarà. Ayacucho, Perù.

2 Asociación Universidad Privada San Juan Bautista-Filial Ica, Perú.

3 Hospital Regional de Ica, Perú.

4 Hospital Santa María del Socorro. Ica, Perú.

A Licenciado en Enfermería.

\section{RESUMEN}

Objetivo: Determinar las condiciones laborales y nivel de estrés en los Enfermeros de un hospital público peruano en el año 2011. Material y métodos: Se realizó un estudio de tipo descriptivo, de corte transversal, la muestra está constituida por 44 enfermeros que se dedican a la labor asistencial, los cuales fueron seleccionados a través del muestreo no probabilístico y por conveniencia de acuerdo a los criterios de inclusión y exclusión. Resultados: Referente a las condiciones laborales son consideradas desfavorables en un $70 \%$ y favorables en un $30 \%$. Respecto al nivel de estrés, el $52 \%$ presenta nivel de estrés medio, el $34 \%$ nivel de estrés bajo y un $14 \%$ presenta estrés alto. Conclusiones: Las condiciones laborales son desfavorables y el nivel de estrés es moderado en los enfermeros de un Hospital público peruano.

Palabras clave: Satisfacción en el trabajo; agotamiento profesional, enfermeros (fuente: DeCS BIREME)

\section{WORKING CONDITIONS AND STRESS LEVEL IN NURSES OF A PUBLIC HOSPITAL PERUVIAN}

\begin{abstract}
To determine the working conditions and level of stress in the nurses of a Peruvian public hospital in 2011.Material and methods:It was realized a descriptive study, and cross-sectional, the sample was composed of 44 nurses who are dedicated to the welfare work, which were selected through the non-probability sampling and by convenience according to the criteria of inclusion and exclusion. Results: Regarding the working conditions are considered unfavourable in a $70 \%$ favorable and 30 $\%$. With regard to the level of stress, the $52 \%$ level of average stress, the $34 \%$ low stress and a $14 \%$ presents high stress. Conclusions: The working conditions are adverse and the stress level is moderate in the nurses in a public hospital peruvian
\end{abstract}

Key words:Job Satisfaction; burnout, professional; nurses (source: MeSH NLM)

\section{INTRODUCCIÓN}

A nivel mundial, la población está siendo afectada por un fenómeno llamado estrés, y ocurre en el momento que se enfrenta a las exigencias del medio, ya sea la vida familiar y la profesional por lo que requiere cierto grado de adaptación y al mismo tiempo ajustar las actividades al tiempo disponible. El personal de enfermería está sometido a múltiples factores estresantes tanto de carácter organizacional como propios de la tarea que ha de realizar, estas características hacen que tenga una incidencia relativamente alta en esta profesión ${ }^{(1)}$.

Según estudios internacionales, los individuos que padecen esta enfermedad, representa un costo de dos millones de dólares al año, ya sea en gastos médicos, internación o simplemente ausencia laboral. Otro dato interesante, es que los "estresados" constituyen el $40 \%$ de la ausencia laboral, reduciendo significativamente el desempeño en sus quehaceres diarios, en un $70 \%{ }^{(2)}$.

Las condiciones laborales en las que se desempeñan los enfermeros en América Latina, promueve que estos profesionales busquen alternativas de desarrollo fuera de su país ${ }^{(3)}$.

En Colombia, el personal de enfermería presenta condiciones particulares de trabajo, representadas por la continuidad de su servicio durante las 24 horas, las diferentes categorías de riesgo presentes en los sitios de trabajo y la característica de género femenino predominante en quienes ejercen la profesión son aspectos que le imprimen una connotación especial, la cual requiere de igual manera, un abordaje particular y participativo, entre la institución y todo el equipo de enfermería ${ }^{(4)}$.

Estudios realizados en Perú con relación a la condición laboral de los trabajadores, los resultados indican mayor incidencia de estrés en el grupo con tiempo de servicio de 1 a 5 años de labor continua en 57,7\%; los que laboran en el turno mañana $83,5 \%$, la frecuencia de 6 horas de labor continua presentó un $57,9 \%$ de casos respectivamente. Al asociar los casos de estrés y morbilidad laboral, se obtuvieron los siguientes diagnósticos: Hipertensión arterial $0,6 \%$, infarto al miocardio $4,9 \%$, otros trastornos cardiovasculares $1,2 \%$, gastritis y/o ulceras pépticas $30,5 \%$, trastornos asmáticos $25,0 \%$ y cuadros de dermatitis $12,2 \%$. 
Con respecto al clima laboral el 75,0\%, del grupo en estudio refirió tener un ambiente inadecuado para el desarrollo de sus actividades, asimismo las actividades inherentes a su trabajo originaban tensión en el 60,4\% y con respecto a las relaciones interpersonales intra-laboral calificó de regular a mala en $59,8 \%$. Asimismo el $72 \%$ consideró su estado de salud actualmente de regular a mala (5).

En tal sentido, el hospital constituye uno de los entornos laborales más estresantes y la profesión de enfermería es considerada una ocupación agotadora y agobiante debido a que diariamente enfrenta situaciones difíciles y riesgosas; sobre todo cuando se encuentran laborando en ambientes inadecuados como es la infraestructura, incluyendo a los factores climáticos y las relaciones interpersonales. El 15 de Agosto del 2007 los peruanos sufrimos uno de los terremotos más devastadores de nuestra historia. Hubo centenares de muertos y miles de damnificados, siendo la población de Ica, una de las más perjudicadas, ésta pérdida invalorable estuvo acompañada de la destrucción y daño de la infraestructura pública básica como escuelas, hospitales, carreteras y acueductos.

A nivel local, en el Hospital Regional de Ica, se observa que las enfermeras están sometidas a un trabajo arduo día a día obviando muchas veces, la importancia de un descanso de mente y cuerpo, por lo que es importante indagar las repercusiones laborales que puedan causar en tales situaciones y cómo repercute en la salud de los enfermeros teniendo en cuenta las exigencias propias de la labor diaria.

\section{MATERIAL Y MÉTODOS}

Se realizó una investigación de enfoque cuantitativo, método descriptivo de corte transversal. La muestra estuvo conformada por 44 enfermeras que se dedican a la labor asistencial. El método de selección de la muestra fue mediante muestreo no probabilístico y por conveniencia. Para la recolección de datos de la investigación se utilizó como técnica la encuesta y como instrumento el cuestionario tipo Escala de Likert estructurado, el cual previamente pasó por un proceso de evaluación a través de un juicio de expertos que comprende la validez y la confiabilidad.

El criterio de confiabilidad del instrumento, se determinó por el coeficiente de Alfa Crombach. Se tuvo en cuenta los principios éticos como el respeto, beneficencia y justicia siendo la participación libre y voluntaria con el permiso necesario y confidencialidad.

\section{RESULTADOS}

El $70 \%$ (31/44) licenciadas tienen edades entre 41 a 50 años, el 25\% (11/44) 51 años a más y el 5\% (2/44) tiene entre 31 a 40 años; el $85 \%$ (37/44) de los encuestados correspondieron al sexo femenino. Con respecto al estado civil el $71 \%(31 / 44)$ son casados y $23 \%$ (10/44) son solteros; el 43\% (19/44) tienen de 11 a 15 años de servicio y el $41 \%$ (18/44) cuentan con más de 15 años de servicios (Tabla 1).

Respecto a las condiciones laborales en el cual se consideró la infraestructura, los factores ambientales así como las relaciones interpersonales, el 70\% (31/44) de los encuestados lo considera como desfavorable y el $30 \%$ $(13 / 44)$ como favorable (Figura 1).

El $52 \%$ (23/44) presenta nivel de estrés medio, el $34 \%$ (15/44) estrés bajo y $14 \%$ (6/44) presenta nivel de estrés alto (Figura 2).

Tabla 1.Datos generales de enfermeras(os) del Hospital Regional Ica, Perú 2011.

\begin{tabular}{lc}
\hline \multicolumn{1}{c}{ Variables } & $\mathbf{N}(\%)$ \\
\hline Edad & \\
$31-40$ Años. & $2(5)$ \\
$41-50$ Años. & $31(70)$ \\
51 a más. & $11(25)$ \\
Sexo & \\
Femenino & $37(85)$ \\
Masculino & $7(15)$ \\
Estado civil & \\
Soltero & $10(23)$ \\
Casado & $31(71)$ \\
Viudo & $2(4)$ \\
Divorciado & $1(2)$ \\
Tiempo de servicio & \\
Menos de 5 años & $1(2)$ \\
De 6 a 10 años & $6(14)$ \\
De 11 a 15 años & $19(43)$ \\
Más de 15 años & $18(41)$ \\
\hline
\end{tabular}

Figura 1. Condiciones laborales de los enfermeros del Hospital Regional de Ica, Julio 2011

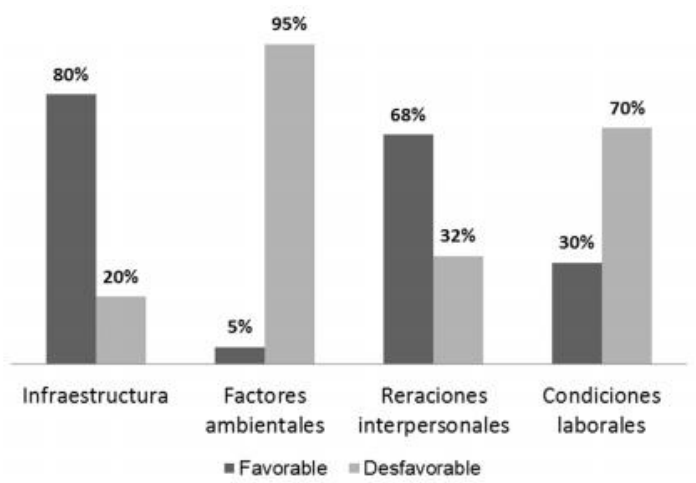

Figura 2. Nivel de estrés de los enfermeros del Hospital Regional de Ica, julio 2011

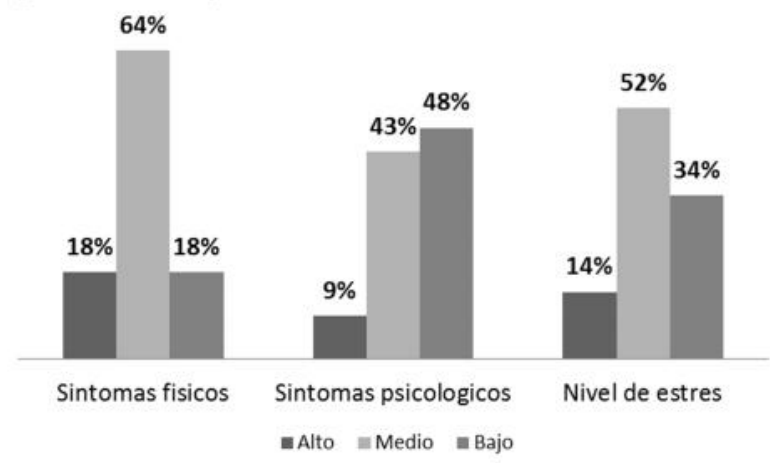




\section{DISCUSIÓN}

La edad, el sexo, el estado civil y el tiempo de servicio son variables que indican determinadas características de comportamiento, de madurez emocional, capacidades físicas e intelectuales; que permite adquirir mayor estabilidad y mayor capacidad para inferir, predomina en nuestro estudio un $70 \%$ de licenciados con edades de 41 a 50 años, donde las personas alcanzan la madurez emocional y se logra el máximo desarrollo de las capacidades y habilidades respecto a la labor que desempeña. El género femenino predominante ha sido como un símbolo para la carrera de enfermería ya que de ellas florece el sentido del cuidado humano por los demás. El estado civil de casado en un $71 \%$ es un factor importante en las organizaciones a tomar en cuenta ya que toda vez que las personas que tienen una conformación adecuada de su familia tiene mayor estabilidad emocional, aspectos personales que lo motivan para trabajar por alcanzar un objetivo.

Los resultados son similares a los obtenidos por Valecillo M. y col. dondeen su mayoría son de género femenino 113 $(89,0 \%)$ y masculino $14(11 \%)$, con edad media de $35,9 \pm$ 8,01 años con un rango de 25 a 61 años, antigüedad promedio de $6,7 \pm 4,2$ años variando entre 5 meses y 31 años $^{(6)}$.

Las condiciones laborales ${ }^{(7)}$ son el conjunto de factores que influyen sobre el bienestar físico y mental de los trabajadores, constituyen el ambiente físico y social del trabajador y los aspectos del contenido del trabajo, que actúan sobre la salud y bienestar de los trabajadores, de un conjunto de variables que al nivel de la sociedad, fijan la duración del tiempo de trabajo, configuran el proceso de trabajo, la organización y división interna del mismo: ritmo, carga física, psíquica y mental del trabajo, en el caso específico de la infraestructura ${ }^{(8)}$.

El tener resultados desfavorables en las condiciones laborales en un $70 \%$, llama la atención ya que se debe contar con ambientes de trabajo sanos y seguros para la salud física, mental e integridad del personal, asimismo contar con los recursos materiales, equipamiento necesario y adecuado para cumplir sus funciones de manera segura y eficaz, que permitan brindar servicios de calidad y que finalmente se refleje en el usuario respecto a la atención de salud.

Unas malas condiciones físicas ambientales ${ }^{(9)}$ pueden ocasionar efectos negativos en la salud que variarán en función de las características de cada persona y su capacidad de aclimatación, así podemos encontrar resfriados, deshidratación, golpes de calor y fatiga, lo que puede incidir en la aparición de accidentes.

Trabajar en un ambiente laboral óptimo ${ }^{(10)}$ es sumamente importante para los empleados, ya que un entorno saludable incide directamente en el desempeño que estos tengan y su bienestar emocional. Juegan un papel muy importante en la organización ${ }^{(11)}$, y en la medida que estas sean satisfactorias se conseguirá un agradable clima laboral, y por ende, contribuirán significativamente al incremento de la productividad.

Un gran porcentaje de la muestra en estudio (52\%), presenta un nivel de estrés medio y un $34 \%$ presenta estrés bajo,siendo estos resultados motivo para que la institución tome medidas con respecto a este tema ya que esto puede llegar a repercutir en la calidad de atención que se brinda al usuario. Estos resultados son similares a los hallazgos de Morales C. ${ }^{(14)}$ donde concluye que 48 $(63,2 \%)$ de enfermeras presenta un nivel de estrés laboral moderado; Coronado $L$. ${ }^{(15)}$ en lo relacionado al nivel de estrés que experimentan los enfermeros concluye que la mayoría del personal de Enfermería estudiado presentan un nivel de estrés medio con ligera tendencia a bajo, que puede traer serias consecuencias de desgaste físico y mental de estos profesionales, que podrían incidir en el deterioro inminente de su salud.Lasconclusiones de Valecillo ${ }^{(6)}$ fueron: los factores físicos ocasionan en un $29,9 \%$ de los trabajadores un estrés medio, en cuanto a los factores psicológicos son generadores de estrés bajo y medio en $26,0 \%$ y $26,8 \%$, respectivamente.

Yonelis F. ${ }^{(12)}$,permite deducir que la mayoría de las enfermeras presentan síntomas físicos durante la jornada laboral, así también presentan síntomas conductuales y se determinó los síntomas afectivos; el 96\% presenta fatiga, de este porcentaje el aspecto más resaltante es la contractura muscular con 61\%; por otro lado, el $57 \%$ padecen de trastorno gástricos, el $48 \%$ padecen de insomnio, en este sentido, el $52 \%$ de las encuestadas reconocieron el bajo rendimiento laboral, en cuanto a la irritabilidad el $87 \%$ de las enfermeras señaló que la frustración incide en el $83 \%$, sin embargo la desmotivación arrojó el $78 \%$, el aspecto pesimismo manifestó $69 \%$, es importante resaltar que en sólo el $30 \%$ de las encuestadas señaló indecisión.

Enfermería es la profesión que requiere un despliegue de actividades que necesita conservar un gran control mental y emocional de mayor rigor, ya que es una carrera expuesta a diferentes situaciones, donde se debe preservar la vida como por ejemplo el manejo del paciente con pronóstico reservado y la necesidad de brindar cuidado, no solamente intensivo, sino también prolongado en el cual también se exige alta concentración y responsabilidad que traen como consecuencia desgaste físico y mental, además de la exigencia de mantener en forma permanente el espíritu de compartir, con el enfermo y su familia, las horas de angustia, depresión y dolor por lo que es indispensable que se establezca estrategias de intervención para prevenir el estrés laboral en los enfermeros de los diferentes servicios tales como reuniones de intercambio de ideas, opiniones, rotación de las enfermeras de las unidades críticas, capacitación periódica sobre estrategias y técnicas para prevenir el estrés lo que fomentará el bienestar y la productividad laboral. 
Fuentes de financiamiento: autofinanciado.

Conflicto de intereses: los autores declaran no tener conflictos de interés.

\section{Correspondencia:}

Olinda Oscco Torres

Correo electrónico: chinita25_40@hotmail.com

\section{REFERENCIAS BIBLIOGRÁFICAS}

1. Navarro GE. Conocimiento y manejo del estrés en enfermería de los servicios de medicina de los hospitales Arzobispo Loayza y Dos de Mayo" Perú - 1996. [Tesis para optar el Título Profesional de Licenciada en Enfermería] U.N.M.S.M. Perú, 1996. 50 pp.

2. Pérez K, Fajardo $\mathbf{M}$. Estrés una enfermedad que cada año suma más adeptos Chile Disponible en :http//periodismoudm.wordpress.com/.../estresuna-enfermedad-que-cada-ano-suma-masadeptos-chile-"pais-de-estresados"

3. Arango G. La globalización como reto para la formación de profesionales de Enfermería en América Latina. Pontificia Universidad Javeriana. Colombia. Avances en enfermería. 2004; 12(2):427.

4. Carvallo B. Revista electrónica de Enfermería. Memorias del I congreso de profesionales de enfermería clínica y VIII simposio: actualizaciones en enfermería. Colombia. Disponible en: www.encolombia.com/.../enfermería/enfermeria630 3-memorias.htm

5. Rivero E.Prevalencia de estrés en trabajadores de salud MINSA de la dirección de salud IV Lima Perú. Dirección de salud IV Lima Este. Direcciñon ejecitiva de salud ambiental. Minsa-Perú. 2005. 18 pp.

6. Valecillo M, Quevedo A, Palma A, Dos Santos A. Síntomas musculo esqueléticos y Estrés laboral en el Personal de Enfermería de un Hospital Militar. Estado de Zulia. Venezuela2009. Salud de los trabajadores 2009; 17(2): 85-95.

7. Arias R. Conceptos decondicionesy medio ambiente de trabajo. OIT 2008.
8. Parra M. Conceptos Básicos de Salud Laboral. Organización Internacional del Trabajo 2003Primera edición 2003. Chile Disponible en: http://www.oitchile.cl/pdf/publicaciones/ser/ser009. $\underline{\mathrm{pdf}}$

9. Martin Daza, F. El estrés: proceso de generación en el ámbito laboral. Nota técnica de prevención Num.318. INSHT- Instituto nacional de salud del trabajo (España) Disponible en: http://internet.mtas-es/Insht/ntp/ntp 318 htm

10. Cristian R. Relaciones Interpersonales 2009 Disponible en http://www. empleochile.universiablogs.net/.../relacionesinterpersonales-en-el-trabajo/.

11. Yáñez R, Arenas M, Ripoll M. El impacto de las relaciones interpersonales en la satisfacción laboral general 2010. Revista de psicología. 2006; 16(2): 193-201.

12. Yonelis F. Estrés laboral y su relación con el síndrome de Burnout. Madrid. España 2005.

13. Tomasina E, Bozzo E, Chaves E, Pucci F. Impacto de las condiciones laborales en la salud de trabajadores de un centro quirúrgico. Sociedad Peruana de Urología. 2007; 34(2): 2-8.

14. Morales C. Nivel de estrés laboral de las enfermeras que laboran en las áreas críticas del Instituto Nacional de salud del niño. [Tesis para optar el título de licenciada en enfermería] Lima: UNMSM. 2006. $106 \mathrm{p}$.

15. Coronado LK. Factores laborales y niveles de estrés laboral en enfermeros de los servicios críticos y medicina del Hospital Nacional Daniel A. Carrión.[Tesis para optar el título de licenciada en enfermería] Lima: UNMSM. 2006. 148 p.

Recibido: 12 / 08 / 2012

Aceptado para publicación: 11 / 12 / 2012 